\title{
Luciferase Reporter Assay System for Deciphering GPCR Pathways
}

\author{
Zhijie Cheng, Denise Garvin, Aileen Paguio, Pete Stecha, Keith Wood and Frank Fan*
}

Department of Research, Promega Corporation, Madison, WI53711, USA

\begin{abstract}
The G protein coupled receptors (GPCR) represent the target class for nearly half of the current therapeutic drugs and remain to be the focus of drug discovery efforts. The complexity of receptor signaling continues to evolve. It is now known that many GPCRs are coupled to multiple G-proteins, which lead to regulation of respective signaling pathways downstream. Deciphering this receptor coupling will aid our understanding of the GPCR function and ultimately developing drug candidates. Here, we report the development of four homogenous bioluminescent reporter assays using improved destabilized luciferases and various response elements: CRE, NFAT-RE, SRE, and SRF-RE. These assays allowed measurement of major GPCR pathways including cAMP production, intracellular $\mathrm{Ca}^{2+}$ mobilizations, ERK/MAPK activity, and small $G$ protein RhoA activity, respectively using the same reporter assay format. We showed that we can decipher $G$ protein activation profiles for exogenous $m_{3}$ muscarinic receptor and endogenous $\beta_{2}$-adrenergic receptors in HEK293 cells by using these four reporter assays. Furthermore, we demonstrated that these assays can be readily used for potency rankings of agonists and antagonists, and for high throughput screening.
\end{abstract}

Keywords: Luciferase, Reporter assays, GPCR, profile of receptor/G protein coupling.

\section{INTRODUCTION}

G protein coupled receptors (GPCRs, or 7TM receptors), constitute the largest superfamily of cell surface receptors and play key roles in regulation of wide variety of physiological processes, including cognition, metabolism, inflammation, immunity and cell proliferation and development [1]. As a result, GPCRs have been popular drug targets, and nearly half of approved drugs on the market directly target GPCRs. Despite the large number of GPCRs that are available as potential drug targets, currently approved drugs only address a small portion of receptors while hundreds are under development. Thus, this target class remains of strong interest for drug discovery.

GPCRs transmit extracellular signals across the plasma membrane via intracellular coupling with heterotrimeric $\mathrm{G}$ proteins. Heterotrimeric $G$ proteins are classified into four subfamilies based on their $G \alpha$ subunit, $G_{s}, G_{i}, G_{q}$ and $G_{12}$. Upon activation of GPCRs, G protein $\alpha$ subunit dissociates from $\beta \gamma$ dimeric subunit, which in turn initiates a cascade of downstream second messenger pathways and eventually induces gene transcription by various response elements including cAMP response element (CRE), nuclear factor of activated T-cells response element (NFAT-RE), serum response element (SRE) and serum response factor response element (SRF-RE, a mutant form of SRE) (Fig. 1). Over the past few decades there have been many substantial discoveries regarding GPCRs structure and function including $\beta$ arrestin-dependent signaling, allosteric modulation, functional selectivity and receptor dimerization that have led to the current understanding of the complexity of receptor signaling [2-4]. Some GPCRs only couple to one type of G protein (e.g., $\mathrm{G}_{\mathrm{s}}$ ), but many GPCRs are known to couple to a

*Address correspondence to this author at the Department of Research, Promega Corporation, Madison, WI53711, USA; Tel: 608-277-2531;

E-mail: frank.fan@promega.com broad range of G-protein families, such as $\mathrm{G}_{\mathrm{i}} / \mathrm{G}_{12}, \mathrm{G}_{\mathrm{q}} / \mathrm{G}_{12}$, or $\mathrm{G}_{\mathrm{i}} / \mathrm{G}_{\mathrm{q}} / \mathrm{G}_{12}$ in a cell type-dependent or compound-specific manner [5]. Current methods employed in GPCR screening programs measure $\mathrm{G}$ protein signaling by determining change in second messengers such as cAMP, inositol trisphosphate $\left(\mathrm{IP}_{3}\right)$, and intracellular $\mathrm{Ca}^{2+}$ mobilizations, which often demands setting up different assay platforms and requires specialized instrumentation for each pathway which could be costly [6]. Plus, HTS-compatible methods for $\mathrm{G}_{12}-\mathrm{RhoA}$ detection is lacking. Thus, despite of the demands of screening assays for multiple functional readouts, the practical execution for drug screening has been slow.

Reporter gene system is a technology where a reporter gene is synthesized in response to activation of a specific signaling cascade of interests, followed by monitoring of the reporter protein expression by its enzymatic activities linked with a variety of colormetric, fluorescent or luminescent read-outs. Due to its inherent sensitivity, large signal dynamics and simplicity to set up, reporter assay platform has been widely used as high throughput homogenous assay for screening GPCR targets linked to cAMP or $\mathrm{Ca}^{2+}$ signaling [7]. Despite the success, some concerns were raised. These include the requirement of a long induction time, false positives and the fact that signal event is distal from receptor activation. These concerns have been addressed in a number of approaches such as the use of destabilized reporters and co-expression of a constitutively expressed internal control $[7,8]$. The high number of false positives due to the fact that the signaling event measured is so distal from receptor activation could be minimized by coexpression of a constitutively expressed internal control reporter [8]. Here, we report the development of a series of homogenous bioluminescent reporter assays using reporter vectors with CRE-, NFAT-RE, SRE-, SRF-RE- built in upstream of destabilized luciferase gene to measure four major GPCR signaling events: change in cAMP level, $\mathrm{Ca}^{2+}$ mobilization, ERK/MAPK activity and 


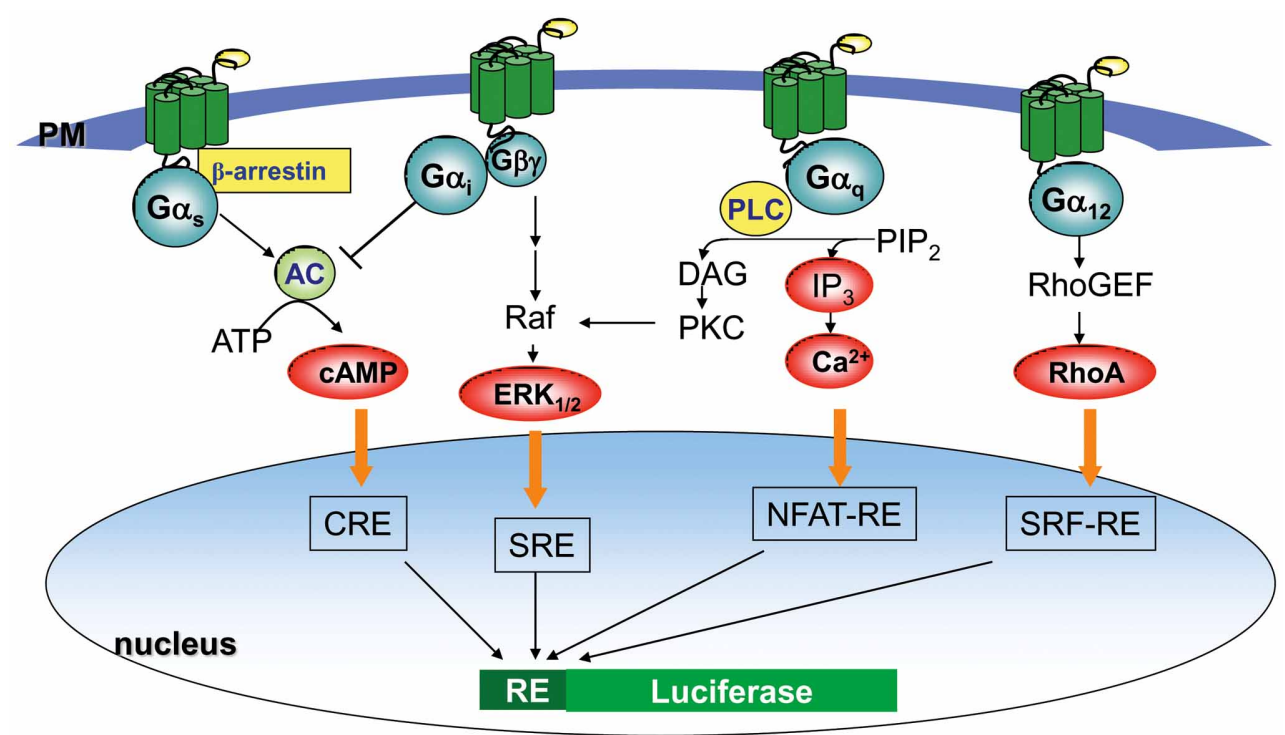

Fig. (1). Schematic diagram showing major GPCR signaling pathways. Upon stimulation, $\mathrm{G}_{\mathrm{s}}$ coupled receptors activate adenylyl cyclase (AC) resulting in an increase of cAMP; $\mathrm{G}_{\mathrm{i}}$-coupled receptors inhibit $A C$, and the $\beta \gamma$ subunits activate ERK pathway; $\mathrm{G}_{\mathrm{q}}$ coupled receptors activate phospholipase $\mathrm{C}$ (PLC) to produce inositol trisphosphate $\left(\mathrm{IP}_{3}\right)$ and diacylglycerol (DAG), which in turn increases intracellular calcium concentration $\left(\mathrm{Ca}^{2+}\right) ; \mathrm{G}_{12}$-coupled receptors activate small $\mathrm{G}$ protein RhoA GTPase.

small $\mathrm{G}$ protein RhoA activation. By using various reporter vectors together, we can measure receptor/G protein coupling profiles for individual receptors, and further compare the potency rankings of various compounds for the same receptor to activate multiple signal pathways. Thus, luciferase reporter assay provides an efficient approach to decipher major GPCR pathways in one simple assay format, which can be used to develop robust HTS assays for drug discovery.

\section{MATERIALS AND METHODOLOGY}

\section{Materials}

pGL4.26[luc2/Hygro], pGL4.27[luc2P/Hygro], pGL4.28 [luc2 CP/Hygro], pGL4.29[CRE/minP/luc2P], pGL4.30 [NFAT-RE/ minP/luc2P], and pF9A CMV hRluc-neo Flexi vectors, and PMA were from Promega (Madison, WI). All other reagents were from Sigma (St. Louis, MO).

\section{Plasmid Construction}

The DNA sequences of five copies of the SRE or SRFRE were synthesized by Integrated DNA Technologies, Inc. (Coralville, Iowa). SRE- and SRF-RE- reporter were constructed by insertion of SRE or SRF-RE into pGL4.26, pGL4.27, or pGL4.28 using Hind III and $B g l$ II restriction enzyme sites. The resulting constructs from pGL4.27 were named as pGL4.33 [SRE/luc2P] and pGL4.34[SRF$\mathrm{RE} /$ luc $2 P$ ], respectively.

The human dopamine D1 receptor (accession number NM_000794) was obtained from American Type Culture Collection (Manassas, VA). The $\mathrm{m}_{3}$ muscarinic receptor (NM_000740.2) was acquired from Origene (Rockville, $\mathrm{MD})$. The coding sequences of both receptors were subcloned into pF9A CMV hRluc-neo Flexi Vector to make receptor-Renilla fusion proteins. The constructs for $\mathrm{m}_{4}$ mus- carinic receptor were from UMR cDNA Resource Center (Rolla, MO). All sequences were verified by restriction digest and sequencing.

\section{Cell Culture, Transfection and Generation of Stable HEK293 Cell Lines}

HEK293 cells were cultured in DMEM (Life Technologies) supplemented with $10 \%$ fetal bovine serum at $37^{\circ} \mathrm{C}$ with a humidified atmosphere at 5\% $\mathrm{CO} 2$. Transient tranfections were done using TransIT®-LT1 Transfection Reagent following manufacturer's protocol (Mirus Bio). Cells were directly plated and transfected in 96-well plate, or plated and transfected in T75 flask, then plated into 384-well plate 4-6 hours after transfection. The cells were changed to serumstarving medium 4-6 hours after transfection and assayed 20 hours later for SRE- or SRF-RE- reporter assay, or assayed 24 hours after transfection for CRE- or NFAT-RE- reporter assay.

Stable HEK293 cell lines expressing reporter vectors (CRE-, NFAT-RE, SRE-, SRF-RE-) were generated by transient transfection followed by hygromycin selection and limiting dilution cloning. Double stable cell lines co-expressing reporter vector and receptor $\left(D_{1}\right.$ dopamine receptor and $m_{3}$ muscarinic receptor) were generated by transfection of receptor construct into single stable line expressing corresponding reporter vector. Cells were plated in complete medium for CRE- or NFAT-RE- assay, or plated in DMEM plus $2 \%$ charcoal/dextran treated FBS (Thermo Scientific) for SRE- or SRF-RE- assay 24 hours before assay.

\section{Luciferase Reporter Assays}

Firefly and renilla luciferase activities, as indicated by relative luminescence units (RLU) were determined using One-Glo or Dual-Glo luciferase assay kits (Promega) according to the manufacturer's instructions. For agonist, fold of 
induction $=$ firefly $\mathrm{RLU}_{\text {induced }} /$ firefly $\mathrm{RLU}_{\text {uninduced }}$. For antagonist, $\%$ of control $=100 \times$ firefly $\mathrm{RLU}_{\text {(agonist+antagonist) }} /$ fire-

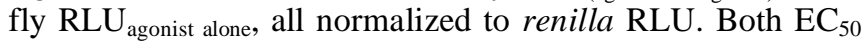
and $\mathrm{IC}_{50}$ values were generated using GraphPad Prism software. $Z^{\prime}$ values were determined as $Z^{\prime}=1-\left[\left(3 \times S_{\text {inudced }}+\right.\right.$ $\left.3 \times \mathrm{SD}_{\text {uninduced }}\right) /\left(\right.$ average $_{\text {induced }}-$ average $\left.\left._{\text {uninduced }}\right)\right]$.

\section{RESULTS AND DISCUSSION}

\section{Improved Assay Performance by Using Destabilized Lu- ciferase Reporter}

Previously, we have shown that the luciferase reporter vectors (pGL4 series) containing minimal promoter and destabilized luciferase gene improve the responsiveness of

A.

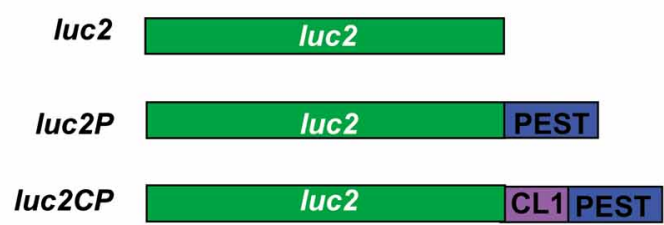

B.

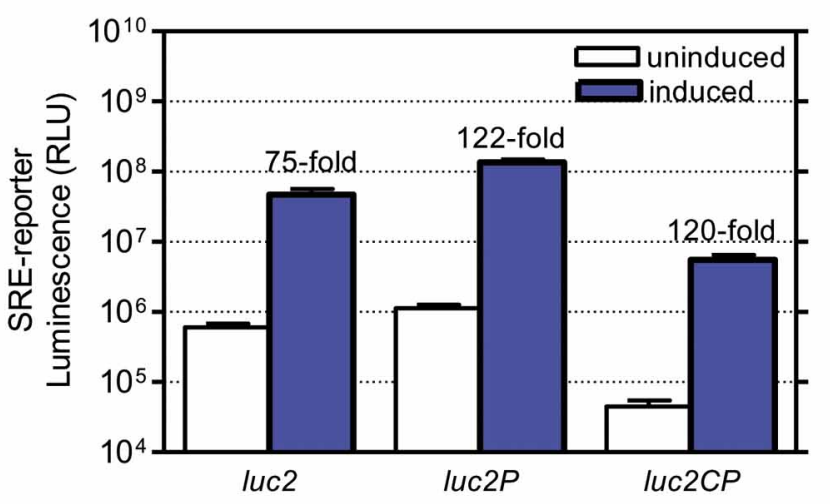

D.

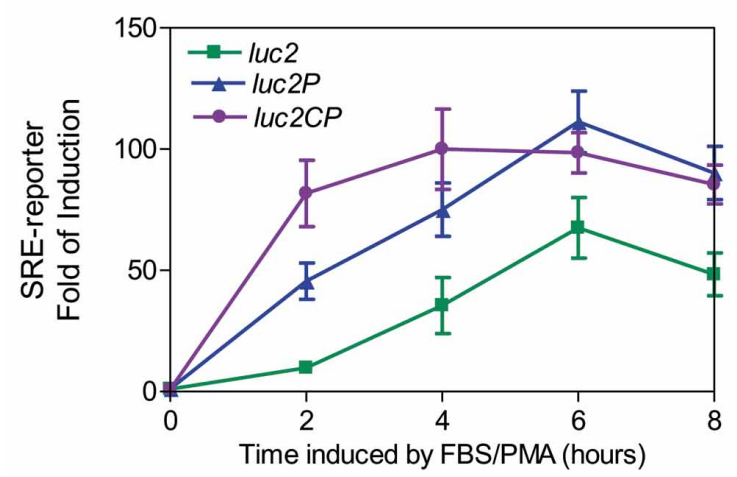

cAMP response element to $G_{s}$ coupled receptor [8]. To evaluate if this is also applicable for other response elements which are specific for different signal pathways, we constructed SRE- and SRF-RE- reporter in various pGL4 vectors containing luciferase gene with or without protein degradation sequence hPEST (Pro-Glu-Ser-Thr) and CL1 [9, 10] as shown in Fig. (2A). SRE is known to respond to ternary complex factor (TCF)-dependent ERK/MAPK pathway, while SRF-RE, a mutant form of SRE lacking TCF binding domain, is newly designed to respond to SRF-dependent and TCF-independent pathway such as RhoA activation [11]. GPCRs, particularly those coupled with $G_{i}$ and $G_{q}$ activate ERK/MAPK pathway and induce transcriptional activation

C.

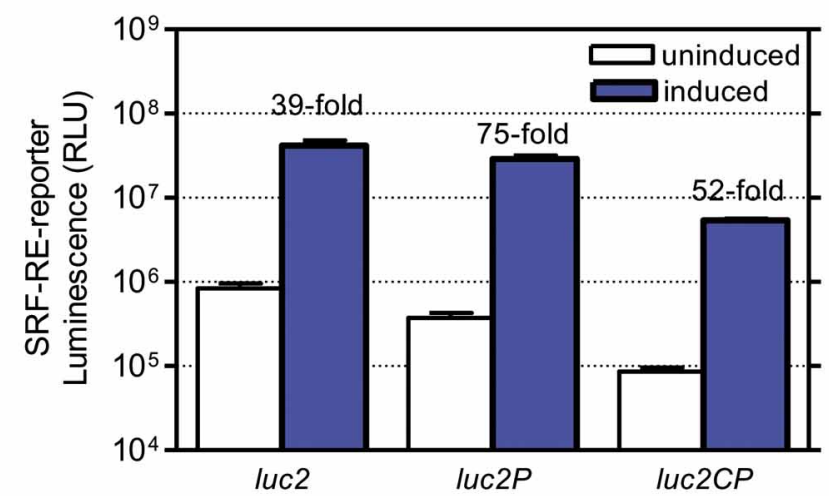

E.

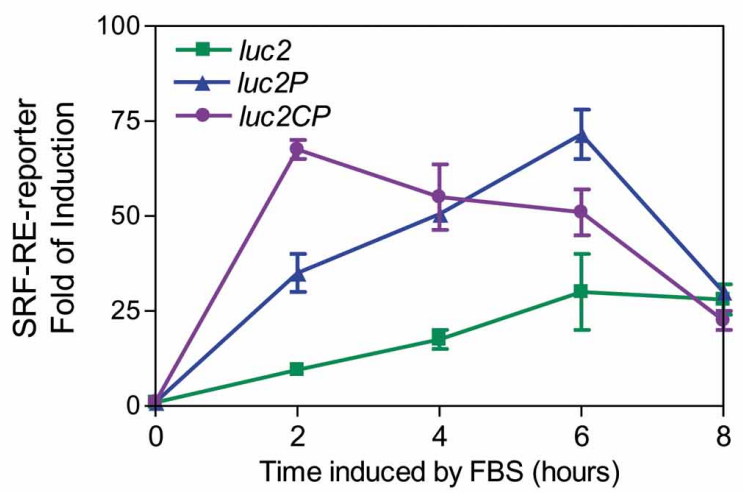

Fig. (2). Destabilized luciferase genes increase the reporter response and reduce the assay time to reach maximum induction. luc2, firefly luciferase; luc $2 P$, firefly luciferase with hPEST sequence; luc $2 C P$, firefly luciferase with hCL1 and hPEST sequences. HEK293 cells transiently expressing various versions of SRE- or SRF-RE- reporters $(l u c 2$, luc2P or $l u c 2 C P$ ) were serum starved, induced with $20 \%$ FBS plus 10ng/ml PMA for SRE (2B, 2D), or with 20\% FBS for SRF-RE (2C, 2E) for 2-8 hours. Cells not induced after serum starvation were used as control (uninduced). Firefly luciferase activity (RLU) were determined using One-Glo assay system and measured on the GloMax 96 multiplate luminometer. (2B, 2C) Firefly luciferase activity (RLU) and fold of induction measured after six hours of induction. (2D, 2E) Time course of fold of induction of SRE- and SRF-RE measured every two hours for 8 hours after induction. 
of SRE, while GPCRs coupled with $\mathrm{G}_{12}$ family are known to activate Rho guanine nucleotide exchange factors (RhoGEFs) which leads to activation of RhoA and transcriptional activation of SRF-RE [12]. This is particularly important with increasing interests in HTS drug screening targeting $\mathrm{G}_{12} /$ RhoA pathway while there are no HTS-compatible methods available for $\mathrm{G}_{12} /$ RhoA so far.

SRE-mediated gene transcription is known to be rapidly induced by serum, phorbol esters and growth factors in TCF and SRF-dependent manner, while SRF alone can mediate transcription activation of SRF-RE by serum mostly through the binding to cell surface receptors of the mitogenic agents such as LPA in serum $[11,12]$. Thus, to establish our assay we first used serum plus PMA to stimulate SRE reporter, and used serum alone to simulate SRF-RE reporter. When various SRE- or SRF-RE reporters were transiently transfected into HEK293 cells, the luciferase reporters containing protein degradation sequence hPEST (luc $2 P$ ), or both hPEST and CL1 (luc2CP) showed 5-10 times lower assay background due to decreased basal expression level of reporter proteins (Fig. 2B, 2C). The cells were further induced with FBS plus PMA (for SRE-) or FBS alone (for SRF-RE-) for different time points up to 24 hours (data not shown for $8+$

A.

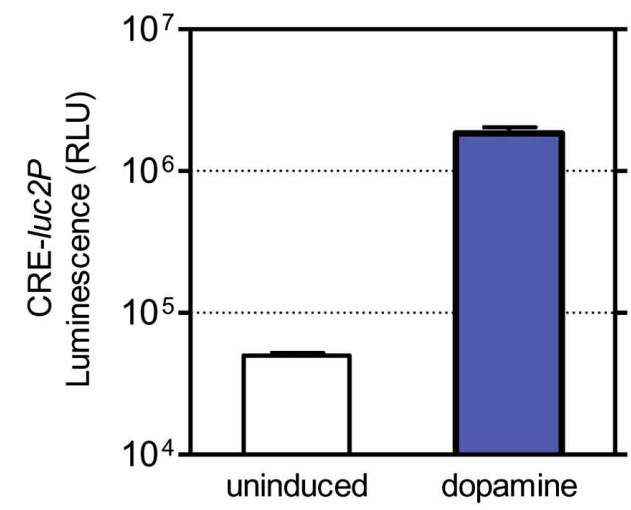

C.

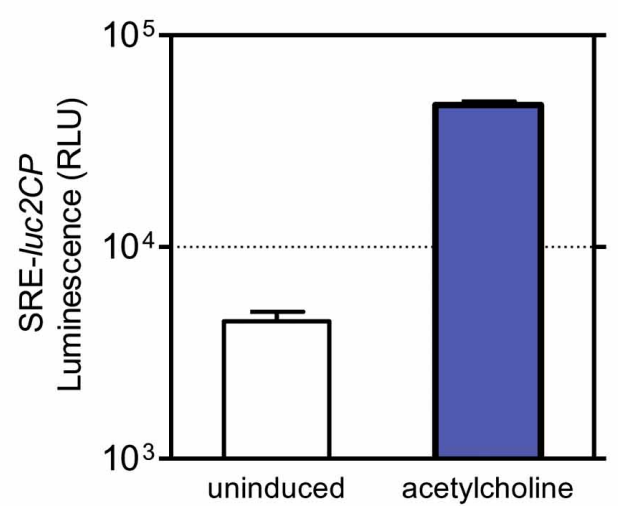

hour time point). As shown in Fig. (2D and 2E), the destabilized SRE- and SRF-RE- reporters reached the maximum of response in a shorter time (2-6 hours) than traditional luciferase gene ( $8+$ hours).

The long incubation time required by traditional reporter assays has limited the application of reporter technology in HTS drug screening due to the concerns of cytotoxicity by the compounds. Therefore, deployment of the destabilized reporter gene will remove this hurdle with improved response dynamics and reduced assay time (2-6 hours) which in turn could potentially minimize secondary effects (such as toxicity effect) arising from the prolonged incubation of cells with chemical compounds. Here we have shown that $L u c 2 P$ versions of SRE and SRF-RE reached the peak response in six hours (Fig. 2D, 2E) with minimal sacrifice of basal reporter expression (Fig. 2B, 2C). Similar results were observed when we further measured several GPCR responses by comparing these SRE- or SRF-RE reporters with or without protein degradation sequences (data not shown), confirming the potentials of using destabilized luciferase reporters in GPCR drug screening. Thus, in consideration of both assay dynamics and signal output, we chose the reporter vectors with protein degradation sequence hPEST incorporated

B.

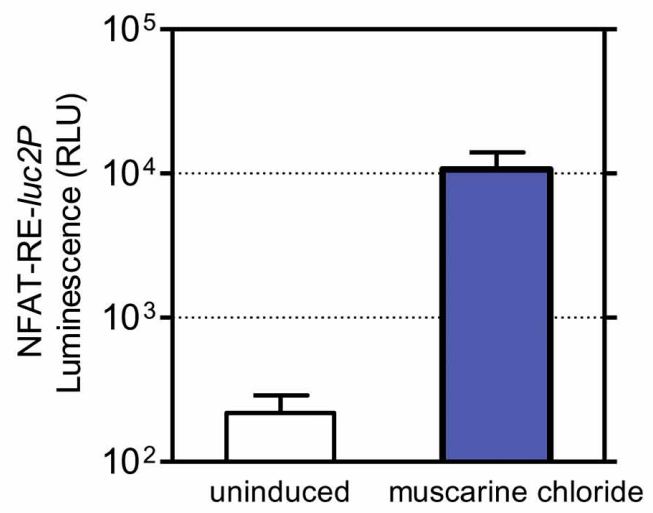

D.

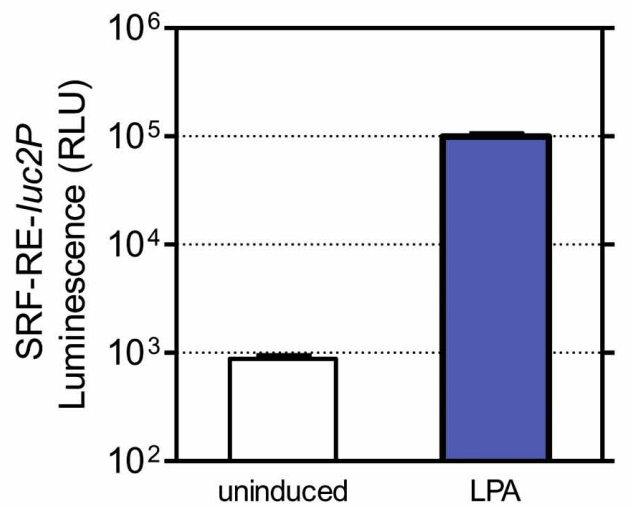

Fig. (3). GPCR assays for $G_{s}, G_{i}, G_{q}$, and $G_{12}$ coupled receptors in 384-well format. HEK293 cells used are either stably expressing $D_{1}$ dopamine receptor and CRE-luc2P $(\mathbf{A}), \mathrm{m}_{3}$ muscarinic receptor with NFAT-RE-luc $2 P$ reporter $(\mathbf{B})$, SRF-RE-luc $2 P$ reporter (test endogenous EDG receptor) (D), or transiently expressing $\mathrm{m}_{4}$ muscarinic receptor with SRE-luc2CP reporter (C). Cells were induced with various agonists in 384-well format. Agonists used (time of induction): $1 \mu \mathrm{M}$ dopamine (4 hours); $1 \mu \mathrm{M}$ muscarine chloride (8 hours); $10 \mu \mathrm{M}$ acetylcholine ( 2 hours); $10 \mu \mathrm{M}$ LPA (6 hours); Firefly luciferase activity was measured using One-Glo assay system. Fold of induction and Z' values were determined as described in "Materials and Methodology". 
Table 1. Summary of Fold Change and Z' Value of Four Different Receptors Known to Couple with Different G Protein Pathways, Measured by Individual Luciferase Reporter Assays

\begin{tabular}{|c|c|c|c|c|}
\hline $\begin{array}{c}\text { Luciferase } \\
\text { Reporter }\end{array}$ & Receptor & $\begin{array}{c}\text { G-protein } \\
\text { subunit }\end{array}$ & $\begin{array}{c}\text { Fold } \\
\text { change }\end{array}$ & $\begin{array}{c}\text { Z' in } \\
\text { 384-well }\end{array}$ \\
\hline \hline CRE-luc2P & $\mathrm{DRD}_{1}$ & $\mathrm{G} \alpha_{\mathrm{s}}$ & 366 & 0.78 \\
\hline NFAT-RE-luc2P & $\mathrm{M}_{3} \mathrm{R}$ & $\mathrm{G} \alpha_{\mathrm{q}}$ & 49 & 0.79 \\
\hline SRE-luc2CP & $\mathrm{M}_{4} \mathrm{R}$ & $\mathrm{G} \alpha_{\mathrm{i}}$ & 11 & 0.85 \\
\hline SRF-RE-luc2P & $\mathrm{EDG}$ receptor & $\mathrm{G} \alpha_{12}$ & 127 \\
\hline
\end{tabular}

with luciferase, SRE-luc $2 P$ and SRF-RE-luc $2 P$ and six hour induction time for most of the following evaluation, unless otherwise indicated.

\section{Amenable for High Throughput Screening}

To determine if luciferase reporter assay is amenable for high-throughput screening for GPCR modulators linked with all major $G$ protein signaling, we chose four individual receptors including $\mathrm{D}_{1}$ dopamine receptor, $\mathrm{m}_{4}$ muscarinic receptor, $\mathrm{m}_{3}$ muscarinic receptor and endogenous EDG receptor, which are known to couple with $\mathrm{G}_{\mathrm{s}}, \mathrm{G}_{\mathrm{i}}, \mathrm{G}_{\mathrm{q}}$ and $\mathrm{G}_{12}$ subfamily respectively, and co-expressed them with the corresponding reporters. In the cases of $G_{s}, G_{q}$ and $G_{12}$ signaling,

A.

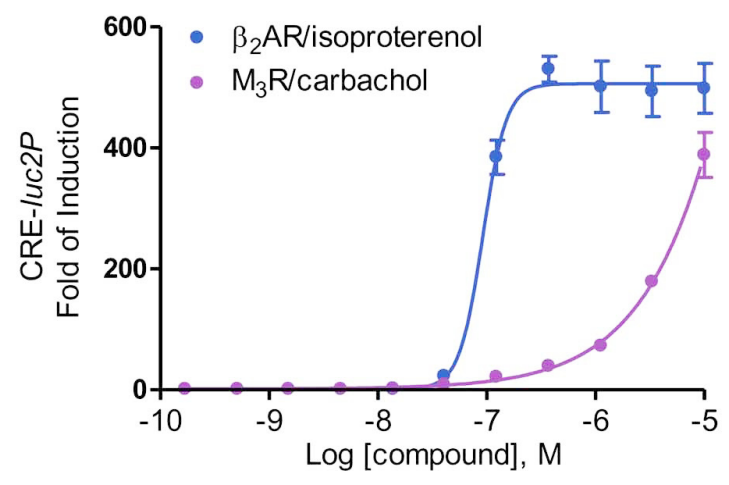

C.

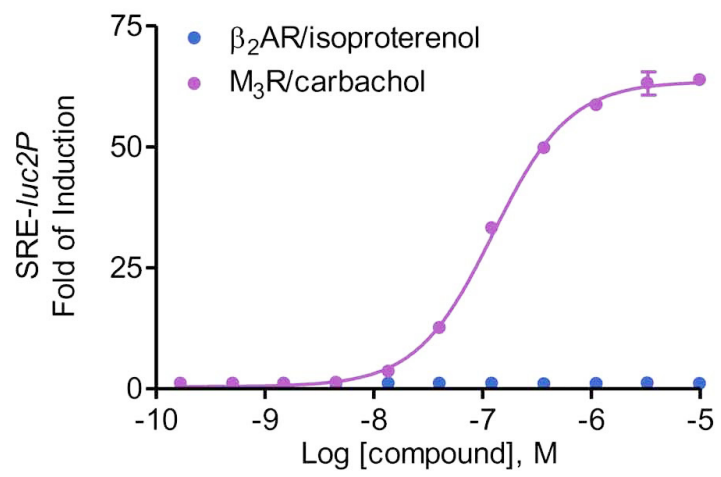

$l u c 2 P$ version of reporters (CRE-luc $2 P$, NFAT-RE-luc $2 P$, SRF-RE-luc $2 P$ ) were tested and shown to give maximal assay performance, while $l u c 2 C P$ of SRE reporter was used for detection of $G_{i} \beta \gamma$-linked MAP kinase activation in order to achieve optimized assay dynamics (Fig. 3, Table 1).

Cells expressing luciferase reporter and receptors were induced in a 384-well plate with various agonists for two to eight hours depending on the requirement of the response elements, RLUs were measured and Z'-factor values were then determined. Z'-factor is one of the major statistical parameters commonly used to evaluate assay performance in high throughput screening. According to the formula, the closer the $\mathrm{Z}$ '-factor is to 1 , the better the assay quality is. In

B.
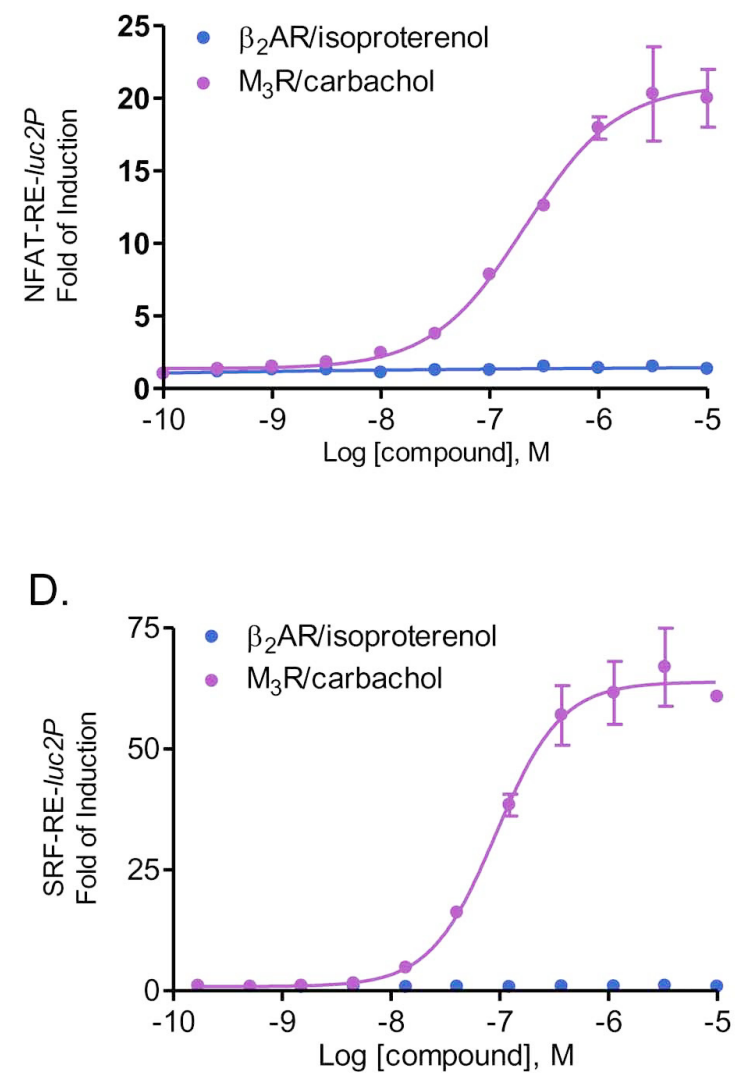

Fig. (4). Profiling of receptor/G protein coupling. HEK293 cells transiently expressing various luciferase reporters as indicated: CRE-luc2P (A), NFAT-RE-luc2P (B), SRE-luc2P (C) or SRF-RE-luc2P (D) were induced with carbachol (for exogenously introduced $\mathrm{m}_{3}$ muscarinic receptor) or isoproterenol (for endogenous $\beta_{2}$-adrenergic receptor) for six hours in 96-well format. Firefly luciferase activity was measured using One-Glo assay system. 
Table 2. Comparison of $\mathbf{E C}_{50}$ of Agonist-Induced Activation of $\mathbf{m}_{3}$ Muscarinic Receptor or of Endogenous $\beta_{2}$ Adrenergic Receptor Measured by Four Luciferase Reporter Assays

\begin{tabular}{|c|c|c|}
\hline $\mathbf{R E}$ & $\mathbf{M}_{\mathbf{3}} \mathbf{R}(\mathbf{M})$ & $\boldsymbol{\beta}_{\mathbf{2}} \mathbf{A R}(\mathbf{M})$ \\
\hline \hline CRE & $>1.6 \times 10^{-4}$ & $9.2 \times 10^{-8}$ \\
\hline NFAT-RE & $8.0 \times 10^{-8}$ & N/A \\
\hline SRE & $1.2 \times 10^{-7}$ & N/A \\
\hline SRF-RE & $9.2 \times 10^{-8}$ & N/A \\
\hline
\end{tabular}

general, Z' values greater than 0.5 are acceptable for HTS [13]. As shown in Table 1, all four reporter assays showed Z'-factor values significantly higher than 0.5 in 384-well format with large response dynamic (fold of induction) range from 10 to 300 fold. Thus, luciferase reporter assays are robust, and can be used for high throughput screening of modulators for all major $\mathrm{G}$ protein pathways.

\section{Deciphering GPCR Coupled Signaling Pathways}

We next evaluate if luciferase reporter assay can be used to profile receptor/G protein coupling in HEK293 cells. It is known that the $m_{3}$ muscarinic receptor, when exogenously

A.

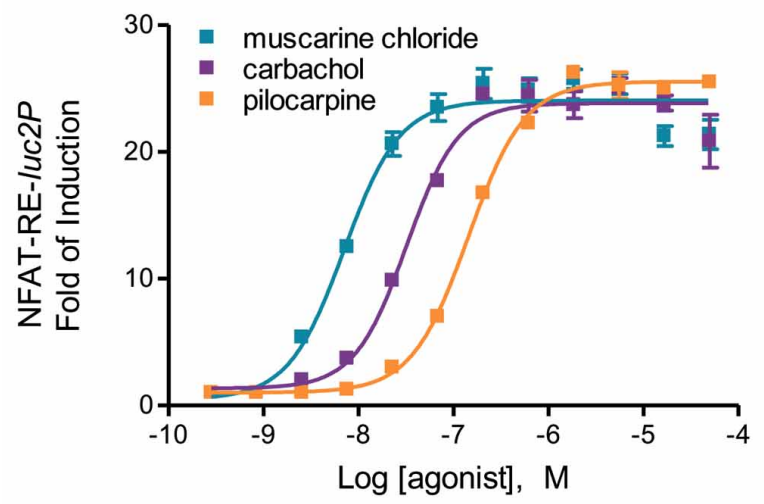

C.

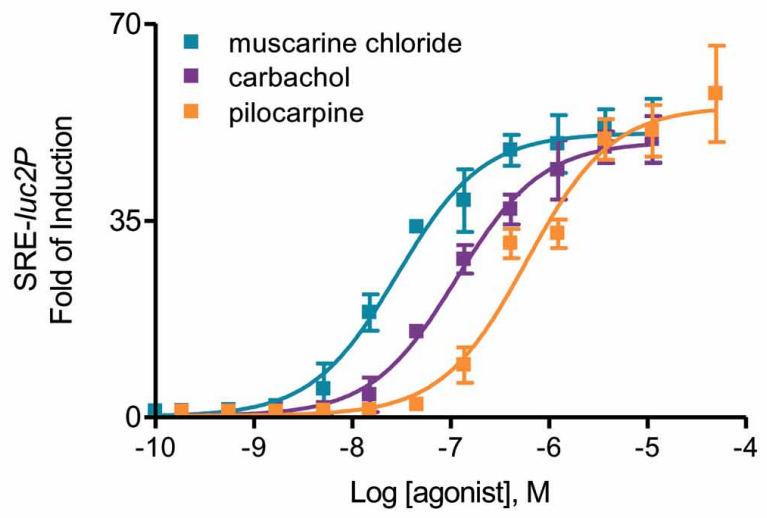

expressed, couples with both $\mathrm{G}_{\mathrm{q}}$ and $\mathrm{G}_{12}$ pathways, while endogenously expressed $\beta_{2}$-adrenergic receptors mostly activate $\mathrm{G}_{\mathrm{s}}$ protein. When we overexpressed $\mathrm{m} 3$ muscarinic receptor in cells in four individual reporter assays, receptor activation by agonist carbachol induced dose-dependent expression of luciferase with distinguishable potencies (Fig. 4). As indicated in Table 2, NFAT-RE-, SRE- and SRF-RE assays showed similar $\mathrm{EC}_{50}$ values by carbachol stimulation, suggesting that activation of $\mathrm{m}_{3}$ muscarinic receptors leads to increase of intracellular calcium concentration, MAP kinase and RhoA activation with similar efficacies. In contrast, $\mathrm{EC}_{50}$ values shown by CRE- reporter assay was almost three logs higher than those by other reporter assays tested, indicating that $m_{3}$ muscarinic receptors activate cAMP production in a much less efficient manner. In contrast, activation of $\beta_{2}$-adrenergic receptors by isoproterenol showed dosedependent response in CRE-reporter assay, but not in NFATRE-, SRE- and SRF-RE- reporter assays, confirming that the major downstream signaling for $\beta_{2}$-adrenergic receptors is cAMP pathway via $\mathrm{G}_{\mathrm{s}}$ protein. Thus, luciferase reporter technology is able to study $\mathrm{G}$ protein activation profiles by the same receptor in one single assay format.

Many GPCRs activate a variety of signaling pathways via distinct $\mathrm{G}$ proteins in cell type dependent manner, which is crucial for their precise regulation of tissue-specific physiological functions [14]. Unfortunately many of the current available screening methods have limitations and are

B.

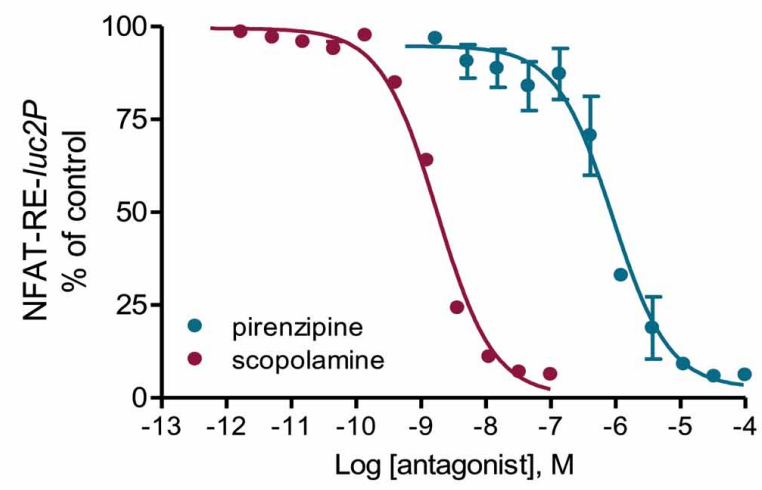

D.

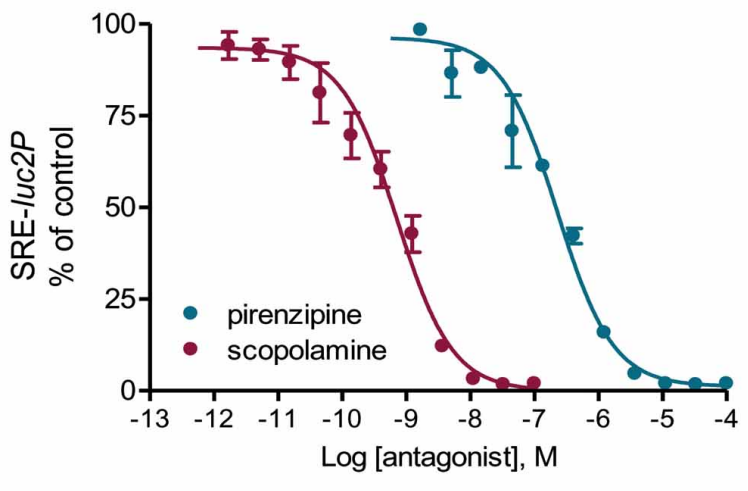

Fig. (5). Potency ranking of agonists and antagonists for $m_{3}$ muscarinic receptor using NFAT-RE- and SRE- reporters. HEK293 cells were transiently transfected with NFAT-RE-luc $2 P(\mathbf{A}, \mathbf{B})$ or SRE-luc $2 P(\mathbf{C}, \mathbf{D})$ along with $\mathrm{m}_{3}$ muscarinic receptor $\left(\mathrm{M}_{3} \mathrm{R}\right)$-Renilla fusion for twenty-four hours. Firefly luciferase activity was measured after 6 hours induction with addition of 1:3 serial dilutions of various agonists $(\mathbf{A}, \mathbf{C})$ or antagonists in the presence of $100 \mathrm{nM}$ muscarine chloride (B, D) using the Dual-Glo assay system in 96-well format. Fold of induction and \% of control were determined as described in "Materials and Methodology". 
unable to measure multiple GPCR signaling events in one assay format. Also, not all GPCR signaling such as RhoA activity can be measured in HTS format. Thus, despite significant interest in profiling for cell type dependent receptor/G protein coupling, it has been progressed rather slowly in current drug discovery practice, partly due to the burden setting up multiple assay platforms and lack of existing detection tools. The bioluminescent reporter assay using destabilized luciferase is fast, highly sensitive and easy to set up. More importantly, as we have shown here in Fig. (4), bioluminescent reporter assays can measure all major GPCR signaling via $\mathrm{G}_{\mathrm{s}}, \mathrm{G}_{\mathrm{i}}, \mathrm{G}_{\mathrm{q}}$ and $\mathrm{G}_{12}$ pathways in one single assay format with great assay dynamics and $Z$ ' values, thereby they could be potentially used to monitor cell type-dependent signaling via $\mathrm{G}$ protein coupled receptors.

\section{Potency Ranking of GPCR Modulators for Different G Protein Pathways}

Since NFAT-RE- or SRE-luc2P reporters respond nicely to activation of $m_{3}$ muscarinic receptor (Fig. 4), we next compared the potency ranking of a series of well-known compounds for $\mathrm{m}_{3}$ muscarinic receptor using both reporter assays. As expected, co-transfection of either reporter vector with $\mathrm{m}_{3}$ muscarinic receptor showed a large dynamic range of assay response upon agonist stimulation and both reporter assays showed identical orders of potency ranking for the agonists and antagonists we tested (Fig. 5). The order of potency rankings for agonists were: muscarine chloride>carbachol>pilocarpine; The $\mathrm{IC}_{50 \mathrm{~s}}$ for antagonists were ranked as: scopolamine>pirenzipine, in the presence of muscarine chloride. Both potency rankings are consistent with those reported in the literature (Table 3). The potencies of same compound/receptor pairs attained from two reporter assays are all within a half-log range, suggesting none of the compounds showed functional selectivity (Fig. 5).

Table 3. Comparison of $\mathbf{E C}_{50}$ (Agonists) and $\mathbf{I C}_{50}$ (Antagonists) for $m_{3}$ Muscarinic Receptor by NFAT-REand SRE- Luciferase Reporter Assays

\begin{tabular}{|c|c|c|}
\hline agonist & NFAT-RE, EC Ev $_{50}(\mathrm{M})$ & SRE, $\mathrm{EC}_{50}(\mathrm{M})$ \\
\hline muscarine chloride & $8.3 \times 10^{-9}$ & $2.8 \times 10^{-8}$ \\
\hline carbachol & $3.6 \times 10^{-8}$ & $1.1 \times 10^{-7}$ \\
\hline pilocarpine & $2.0 \times 10^{-7}$ & $5.2 \times 10^{-7}$ \\
\hline
\end{tabular}

\begin{tabular}{|c|c|c|}
\hline antagonist & NFAT-RE, IC $_{50}(M)$ & SRE, IC $_{50}(M)$ \\
\hline pirenzipine & $9.0 \times 10^{-7}$ & $2.2 \times 10^{-7}$ \\
\hline scopolamine & $1.8 \times 10^{-9}$ & $7.1 \times 10^{-10}$ \\
\hline
\end{tabular}

More evidence indicates that same receptor could activate various signaling pathways with distinct potencies depending on the modulators which could bind to the receptor orthosterically or allosterically. This new concept offers great potential for developing pathway-specific drugs that increase efficacy and reduce side effects, but at the same time add another dimension in assay complexity to drug discovery. In the case of $m_{3}$ muscarinic receptor, activation of $\mathrm{m}_{3}$ muscarinic receptor increases intracellular $\mathrm{Ca}^{2+}$ mobilization and induces ERK/MAPK pathway at the same time, however, it is also reported that MAPK activity via $\mathrm{m}_{3}$ muscarinic receptor is regulated in a calcium-dependent or independent manner in a variety cell types, suggesting the involvement of distinct signal mechanisms [15]. Therefore, by comparing the potencies of same compound/receptor pairs using multiple reporter vectors which measure different $G$ protein downstream signaling, this approach has great potential in drug screening for GPCR modulators with functional selectivity in one simple assay format.

\section{CONCLUSION}

In this study, we developed four homogenous bioluminescent reporter assays using destabilized luciferase. The improved reporter assays showed larger assay dynamics in shorter assay time than using regular luciferase. Using a model system of exogenous or endogenous receptors in HEK293 cells, we demonstrated that all four major $G$ protein subfamilies and downstream pathways can be studied in one luciferase reporter assay format. Furthermore, these assays can be readily used for potency rankings of GPCR modulators and for high throughput screening. Together, our data suggest that these improved luciferase reporter assays are well suited for GPCR research and drug screening, with potential application in establishing receptor/G protein profiles for any particular receptor and drug screening for pathwayspecific GPCR modulators.

\section{ACKNOWLEDGEMENT}

We would like to thank Kevin Kopish for valuable discussion.

\section{REFERENCES}

[1] Lefkowitz RJ. Seven transmembrane receptors: something old, something new. Acta Physiol (Oxf) 2007; 190: 9-19.

[2] Shenoy SK, Drake MT, Nelson CD, et al. beta-arrestin-dependent, $\mathrm{G}$ protein-independent ERK1/2 activation by the beta ${ }_{2}$ adrenergic receptor. J Biol Chem 2006; 281: 1261-73.

[3] Urban JD, Vargas GA, von Zastrow M, Mailman RB. Aripiprazole has functionally selective actions at dopamine D2 receptormediated signaling pathways. Neuropsychopharmacology 2007; 32: 67-77.

[4] Chan WY, McKinzie DL, Bose S, et al. Allosteric modulation of the muscarinic M4 receptor as an approach to treating schizophrenia. Proc Natl Acad Sci U S A 2008; 105: 10978-83.

[5] Wettschureck N, Offermanns S. Mammalian G proteins and their cell type specific functions. Physiol Rev 2005; 85: 1159-204.

[6] Siehler S. Cell-based assays in GPCR drug discovery. Biotechnol J 2008; 3: 471-83.

[7] Bronstein I, Fortin J, Stanley PE, Stewart GS, Kricka LJ. Chemiluminescent and bioluminescent reporter gene assays. Anal Biochem 1994; 219: 169-81.

[8] Fan F, Wood KV. Bioluminescent assays for high-throughput screening. Assay Drug Dev Technol 2007; 5: 127-36.

[9] Li X, Zhao X, Fang Y, et al. Generation of destabilized green fluorescent protein as a transcription reporter. J Biol Chem 1998; 273: 34970-5.

[10] Gilon T, Chomsky O, Kulka RG. Degradation signals for ubiquitin system proteolysis in Saccharomyces cerevisiae. EMBO J 1998 17: 2759-66.

[11] Hill CS, Wynne J, Treisman R. The Rho family GTPases RhoA, Rac1, and $\mathrm{CDC} 42 \mathrm{Hs}$ regulate transcriptional activation by SRF. Cell 1995; 81: 1159-70.

[12] Hill CS, Treisman R. Differential activation of c-fos promoter elements by serum, lysophosphatidic acid, G proteins and polypeptide growth factors. EMBO J 1995; 14: 5037-47. 
[13] Zhang JH, Chung TD, Oldenburg KR. A Simple Statistical Parameter for Use in Evaluation and Validation of High Throughput Screening Assays. J Biomol Screen 1999; 4: 67-73.

[14] Castaño JP, Martínez-Fuentes AJ, Gutiérrez-Pascual E, et al. Intracellular signaling pathways activated by kisspeptins through
GPR54: do multiple signals underlie function diversity? Peptides 2009; 30: 10-5.

[15] Slack BE. The $m_{3}$ muscarinic acetylcholine receptor is coupled to mitogen-activated protein kinase via protein kinase $\mathrm{C}$ and epidermal growth factor receptor kinase. Biochem J 2000; 348: 381-7.

(C) Cheng et al.; Licensee Bentham Open.

This is an open access article licensed under the terms of the Creative Commons Attribution Non-Commercial License (http://creativecommons.org/licenses/by-nc/3.0/) which permits unrestricted, non-commercial use, distribution and reproduction in any medium, provided the work is properly cited. 